\title{
The orbital period of intermediate polar 1WGA J1958.2+3232
}

\author{
S. V. Zharikov, G. H. Tovmassian, J. Echevarría, and A. A. Cárdenas
}

Observatorio Astronómico Nacional, Instituto de Astronomía, UNAM, 22800, Ensenada, B.C., Mexico*

Received 5 September 2000 / Accepted 6 November 2000

\begin{abstract}
The detection of the orbital period of 4.36 is reported for the new Intermediate Polar 1 WGA J1958.2+3232. The orbital period was derived from time-resolved photometric and spectral observations. We also confirmed the $733 \mathrm{~s}$ spin period of the White Dwarf consistent with the X-ray pulsations and were able to distinguish the beat period in the light curve. Strong modulations with orbital period are detected in the emission lines from spectral observations. They show the presence of a bright hot spot on the edge of the accretion disk. The parameters of this recently discovered Intermediate Polar are determined.
\end{abstract}

Key words. stars: individual: 1 WGA J1958.2+3232 - stars: novae, cataclysmic variables - stars: binaries: close X-rays

\section{Introduction}

Cataclysmic variables (CVs) are close binary systems in which mass is transferred from a red dwarf star that fills its Roche lobe onto a white dwarf (WD). Intermediate polars (or DQ Her systems) are a subclass of magnetic cataclysmic variables with an asynchronously rotating $\left(P_{\text {spin }}<P_{\text {orb }}\right)$ magnetic white dwarf (Patterson 1994; Warner 1995). The accretion flow from the red dwarf star forms an accretion disk around the white dwarf, and this disk is disrupted by the magnetic field close to the white dwarf. Within the magnetospheric radius, the material is channelled towards the magnetic polar regions of the white dwarf (Rosen et al. 1988).

The recently discovered pulsating X-ray source 1 WGA J1958.2+3232 (Israel et al. 1998) was announced as a new Intermediated Polar (IP) by Negueruela et al. (2000) from spectral observations. Strong modulations of this source in X-rays were obtained from ROSAT PSPC $(721 \pm 14 \mathrm{~s})$ and a more accurate value $734 \pm 1 \mathrm{~s}$ from ASCA are presented by Israel et al. (1998) and Israel et al. (1999). Photometric observations of the optical counterpart of 1 WGA J1958.2+3232 exhibited strong optical variations, compatible with the X-ray $(\sim 12 \mathrm{~min})$ period (Uslenghi et al. 2000). This modulation was interpreted as evidence of a spin period of the WD in this close binary system.

In this paper we present the results of new photometric and spectral observations of this system.

\footnotetext{
Send offprint requests to: S. V. Zharikov,

e-mail: zhar@astrosen. unam.mx

* Use for smail PO Box 439027, San Diego, CA, 92143-9027, USA.
}

Table 1. Observations log

\begin{tabular}{lcccc}
\hline \hline $\begin{array}{l}\text { HJD start } \\
\text { day }\end{array}$ & Duration & $\begin{array}{c}\text { Time } \\
\text { of exposure }\end{array}$ & Band & Telescope \\
$2541000+$ & $\min$ & $\mathrm{s}$ & & \\
\hline $759.429^{a}$ & 380 & 120 & $R_{\mathrm{c}}$ & $1.5 \mathrm{~m}$ \\
760.383 & 435 & 120 & $R_{\mathrm{c}}$ & $1.5 \mathrm{~m}$ \\
761.855 & 173 & 700 & $4025-5600 \AA$ & $2.12 \mathrm{~m}$ \\
762.649 & 461 & 700 & $4025-5600 \AA$ & $2.12 \mathrm{~m}$ \\
763.678 & 319 & $700 / 350$ & $4025-5600 \AA$ & $2.12 \mathrm{~m}$ \\
\hline
\end{tabular}

${ }^{a} 2$ August.

\section{Observations}

The CCD photometric and spectral observations of the 1 WGA J1958.2+3232 were carried out on 2-5 August 2000 at the $1.5 \mathrm{~m}$ and $2.12 \mathrm{~m}$ telescopes of the Observatorio Astronomico Nacional, San Pedro Martir of the Institute of Astronomy of UNAM, Mexico. The observations $\log$ is presented in Table 1.

\subsection{Optical photometry}

We obtained $R_{\mathrm{c}}$-band time-resolved photometry of the optical counterpart of 1 WGA J1958.2+3232 during two nights in August 2000 at the $1.5 \mathrm{~m}$ telescope. The telescope was equipped with a $1024 \times 1024$ pixel SITE CCD. The frame was reduced in size to $450 \times 450$ pix for faster read-out. It accommodated the object and at least two comparison stars in the field of view. The exposure times were $120 \mathrm{~s}$, which leads to a time resolution of $169 \mathrm{~s}$, taking into account dead time between readouts. In total the object was monitored during $\sim 13.55 \mathrm{~h}(6.3 \mathrm{~h}$ the first night and $7.25 \mathrm{~h}$ the second). The data reduction was 

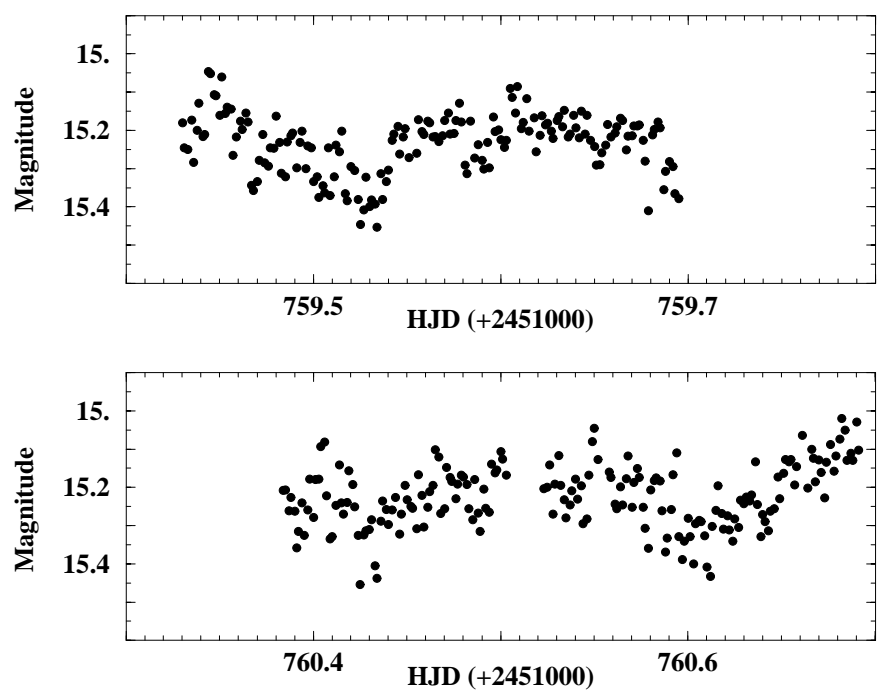

Fig. 1. 1 WGA J1958.2+3232 light curves in $R_{\mathrm{c}}$ band are presented. Binning time is $120 \mathrm{~s}$

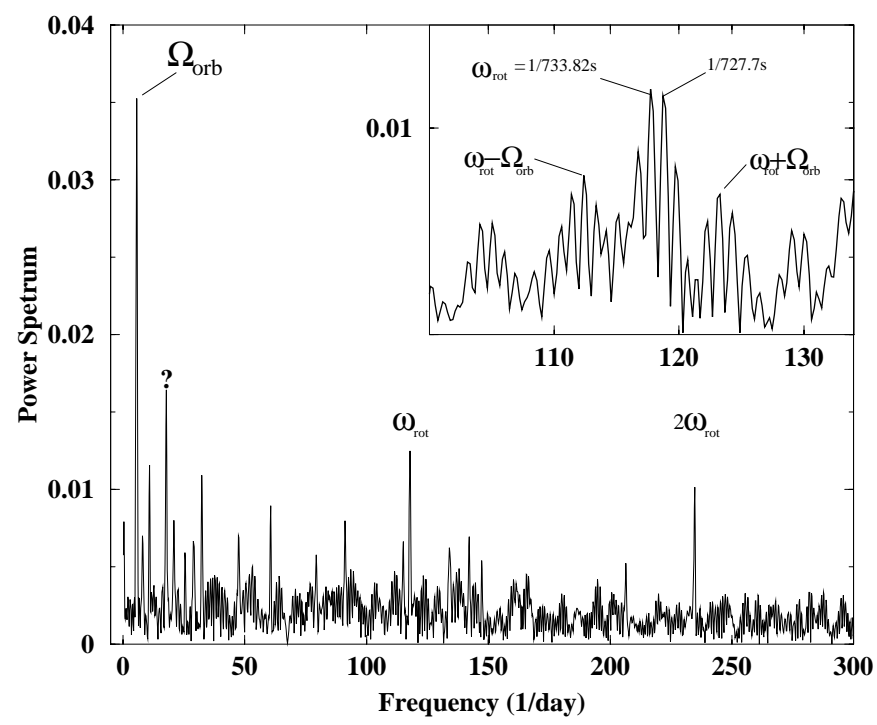

Fig. 2. The CLEANed power spectrum of $R_{\mathrm{c}}$ light curve is given. The orbital period $\Omega_{\text {orb }}$ and $\omega_{\text {rot }}$ spin period frequencies are marked. In the inset part of the cleaned power spectrum with a smaller number of iterations is shown. The beat frequencies $\omega_{\text {rot }}-\Omega_{\text {orb }}, \omega_{\text {rot }}+\Omega_{\text {orb }}$ and $\omega=1 /(727.78 \mathrm{~s})$ are shown

performed by using ESO-MIDAS ${ }^{1}$ and IRAF $^{2}$ software. The images were corrected for bias and flatfield before aperture photometry was carried out. An estimate of the uncertainty of the CCD photometry of the optical coun-

1 ESO-MIDAS is a copyright protected software product of the European Southern Observatory, and provides general tools for image processing and data reduction.

${ }^{2}$ IRAF is the Image Reduction and Analysis Facility, a general purpose software system for the reduction and analysis of astronomical data. IRAF is written and supported by the IRAF programming group at the National Optical Astronomy Observatories (NOAO) in Tucson, Arizona. NOAO is operated by the Association of Universities for Research in Astronomy (AURA), Inc. under cooperative agreement with the National Science Foundation. terpart of 1 WGA J1958.2+3232 was obtained from the dispersion of magnitudes in the differential photometry of comparison stars with similar brightness. The dispersion ranged from 0.005 to $0.01 \mathrm{mag}$. We did not obtain an absolute calibration for our photometric data.

\subsection{Optical spectroscopy}

Time-resolved spectroscopy of the optical counterpart of 1 WGA J1958.2+3232 was obtained on 4-6 Aug., 2000 using the Boller \& Chivens spectrograph installed in the Cassegrain focus of the $2.12 \mathrm{~m}$ telescope. We used the $400 \mathrm{l} / \mathrm{mm}$ grating with a $13^{\circ} 54$ blaze in the second order, combined with the blue BG39 filter and CCD TEK $1024 \times 1024$ pix with a $0.24 \mu$ pixel size. The slit width was 1.5 arcsec projected on the sky. This combination yielded a spectral resolution of $2.7 \AA \mathrm{FWHM}$ and provided a wavelength coverage of $\lambda 4050-5600 \AA$. Of three nights of spectral observations, the second and third nights were disrupted by passing clouds. However the seeing was satisfactory with images $\leq 1.2$ arcsec. The slit was oriented with position angle of $306^{\circ}$ to accommodate a nearby star for the flux level control. The exposure time in the first two nights was $700 \mathrm{~s}$, while on the third night, 700 and $350 \mathrm{~s}$. The He-Ar comparison spectra were taken every $\sim 120$ min. A total of 68 spectra was obtained. The IRAF long slit spectroscopic reduction package was used for extraction of spectra, wavelength and flux calibrations. Beforehand the images were reduced for bias and cosmic rays.

\section{Results}

\subsection{Light curve. Orbital and spin modulations}

The object shows multi-scale time variability with a range of 0.3 magnitudes (see Fig. 1). Four pronounced eclipselike depressions obviously shape the light curve. Strong flickering with optical pulse amplitude (semi-amplitude) of about 0.1 magnitude is also obvious in the light curve detected and identified earlier (Israel et al. 1998; Uslenghi et al. 2000) as spin related modulations. The photometric data of 1 WGA J1958.2+3232 were analyzed for periodicities using the Discrete Fourier Transform (DFT) code (Deeming 1975) with a CLEAN procedure (Roberts et al. 1987). The CLEANed power spectrum (Fig. 2) of $R_{\mathrm{c}}$ photometric data shows a clear peak at $\Omega_{\text {orb }}=5.54996 \pm 0.39624$ ( $1 /$ day $)$, corresponding to $P_{\text {orb }}=$ $0.1802 \pm 0.0065 \mathrm{~d}$. This peak is caused by the above mentioned eclipses in the light curve and clearly marks the orbital period of the system.

We also found a significant peak at the spin period $\omega_{\text {rot }}$ of the WD corresponding to $733.82 \pm 1.25 \mathrm{~s}$. This period is in excellent agreement with that recently discovered by ASCA X-ray pulsations (Israel et al. 1999). The beat frequencies at $\omega_{\text {rot }}-\Omega_{\text {orb }}, \omega_{\text {rot }}+\Omega_{\text {orb }}$ are also present in the CLEANed power spectrum but with a smaller number of iterations (see insert in the upper right corner of the Fig. 2). The harmonics of the basic frequencies $\Omega_{\text {orb }}$ and $\omega_{\text {rot }}$ are detected as well. Besides these, there are 


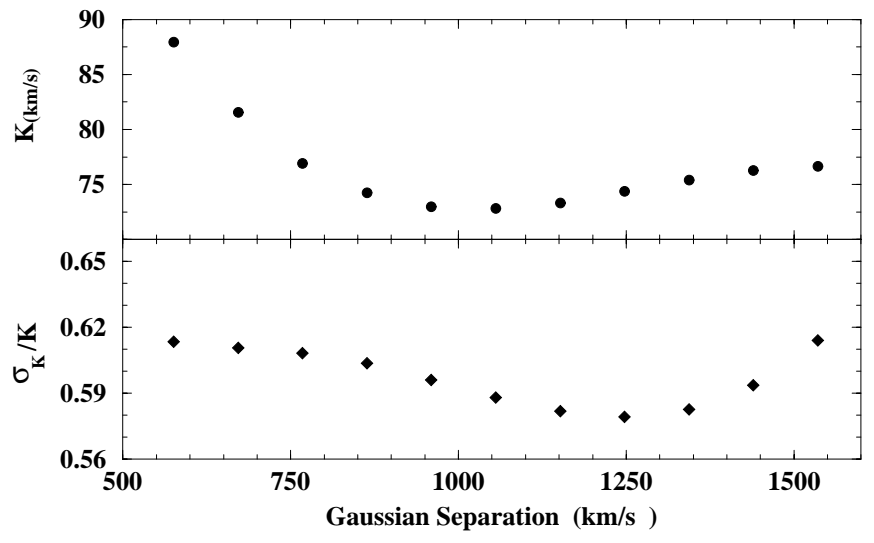

Fig. 3. The diagnostic diagrams for the $\mathrm{H}_{\beta}$ emission lines. The radial velocity semi-amplitude $K$, the ratio $\sigma_{\mathrm{K}} / K$ are plotted as a function of the Gaussian separation, obtained for a period of 0.18152 days

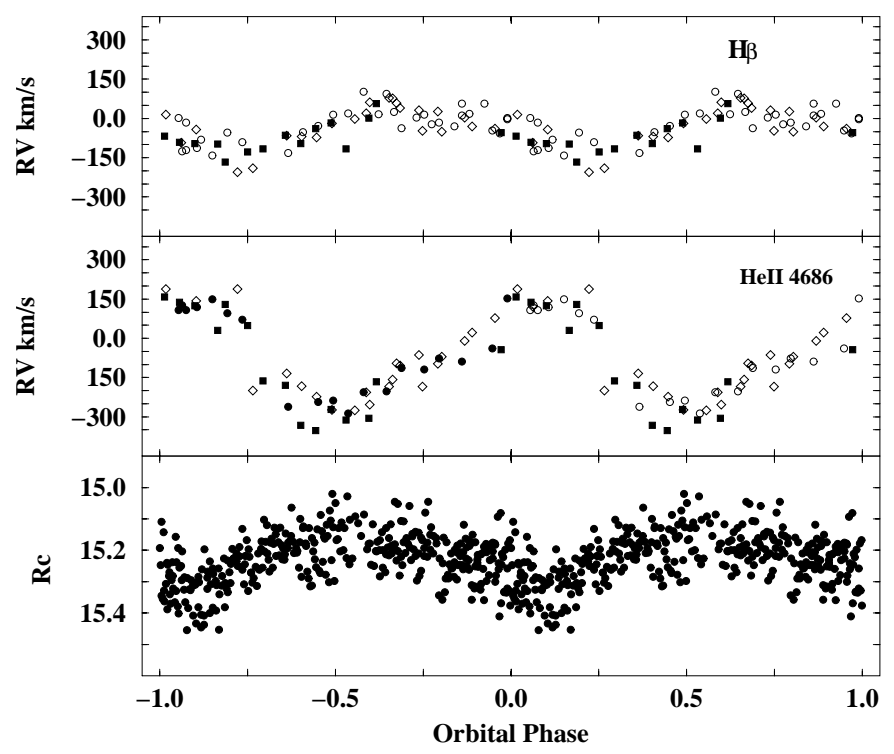

Fig. 4. The radial velocity curves for the emission lines of $\mathrm{H}_{\beta}$ and He II 4686 phased on the spectroscopic orbital period (0.18152 days) are presented upper and middle panels respectively. The filled circles corresponds to 4 Aug., filled rectangulars and open diamonds are 5 and 6 Aug., respectively. Binning time is $700 \mathrm{~s} . R_{\mathrm{c}}$ light curve of 1 WGA J1958.2+3232 is presented in the lower panel. The binning time is $120 \mathrm{~s}$

comparably significant peaks at the periods of $727.78 \mathrm{~s}$ and $1.36 \mathrm{~h}$. The former was detected also by Uslengi et al. (2000) and is probably the one-day alias of $\omega_{\text {rot }}$, while for the latter we could not find any reasonable explanation.

\subsection{Radial velocity variations and binary system parameters}

The spectrum of 1 WGA J1958.2+3232 shows features characteristic for Cataclysmic Variables. We refer to Negueruela et al. (2000), who obtained a spectrum of the object in a wider spectral range and with better spectral resolution. We also obtained time-resolved spectroscopy of 1 WGA J1958.2+3232 around the emission lines of $\mathrm{H}_{\beta}$ and HeII, covering several orbits. Thus, we were able to

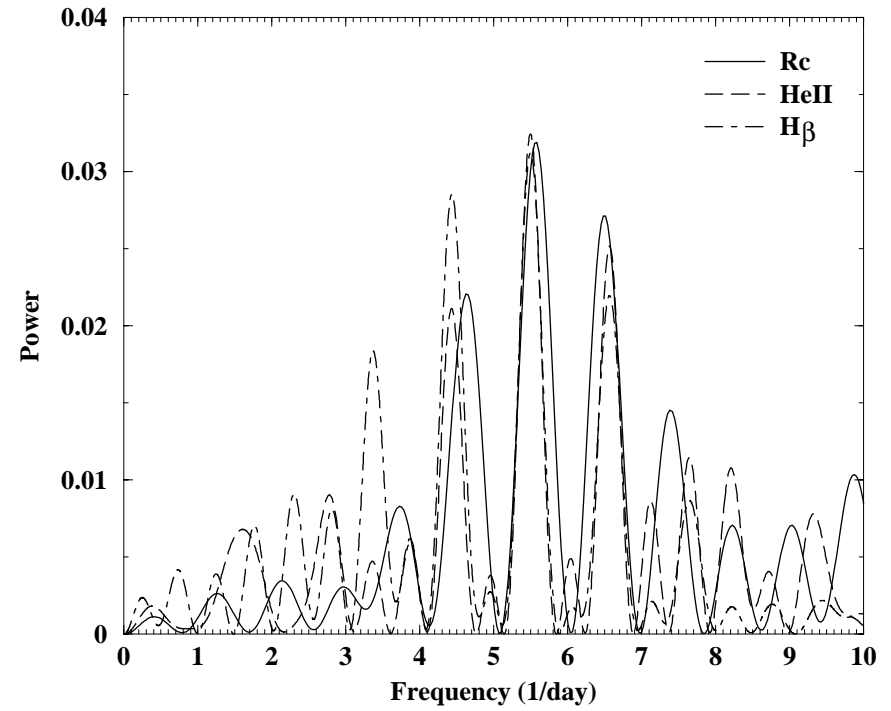

Fig. 5. The power spectrum of the $R_{\mathrm{c}}$ light curve and the He II 4686 and $\mathrm{H}_{\beta}$ radial velocity curves are presented. They are scaled to the amplitude of the $R_{\mathrm{c}}$ power spectrum. The maximum peak of frequency corresponds to the orbital period of the system $P_{\text {orb }}=0.18152 \pm 0.0011 \mathrm{~d}$

examine periodical variations in the spectrum of the object, primarily in the emission lines. The simple stacking of consecutive spectra onto the trailed spectrum showed strong variability in the lines. It is distinct in the Balmer lines and in the higher excitation lines of ionized Helium. The Balmer lines are double-peaked with an S-wave moving inside, which makes it hard to see the periodic pattern. In the He II 4686 line the central narrow component dominates in most of the phases, and it shows clear sinusoidal variation.

In order to determine the orbital elements we measured the radial velocities (RV) of $\mathrm{H}_{\beta}$ applying the double Gaussian deconvolution method introduced by Schneider \& Young (1980), and further developed by Shafter (1983). This method is especially efficient for measurements of the orbital motion of CVs with a prominent spot at the edge of the accretion disk, contaminating the central parts of the emission lines. It allows us to measure RV variations using the wings of the lines. The width of the Gaussians were chosen to be slightly larger than our spectral resolution $(8.5 \AA)$, where deconvolution was reached at all orbital phases. The radial velocities were measured as a function of distance $a$ between the Gaussians, and then the diagnostic diagrams were constructed using an initial guess for the orbital period, derived from photometry and from preliminary radial velocity measurements via Gaussian fits to the lines. The optimal value of separation $\left(a=1175 \mathrm{~km} \mathrm{~s}^{-1}\right)$ was determined from the diagnostic diagrams, and the RV values measured for these Gaussian separations were again subjected to a power spectrum analysis in order to refine the period. The spectroscopic period peaked at a slightly longer value, than the photometric period (however within the errors of the photometric period). This method quickly converged and after 


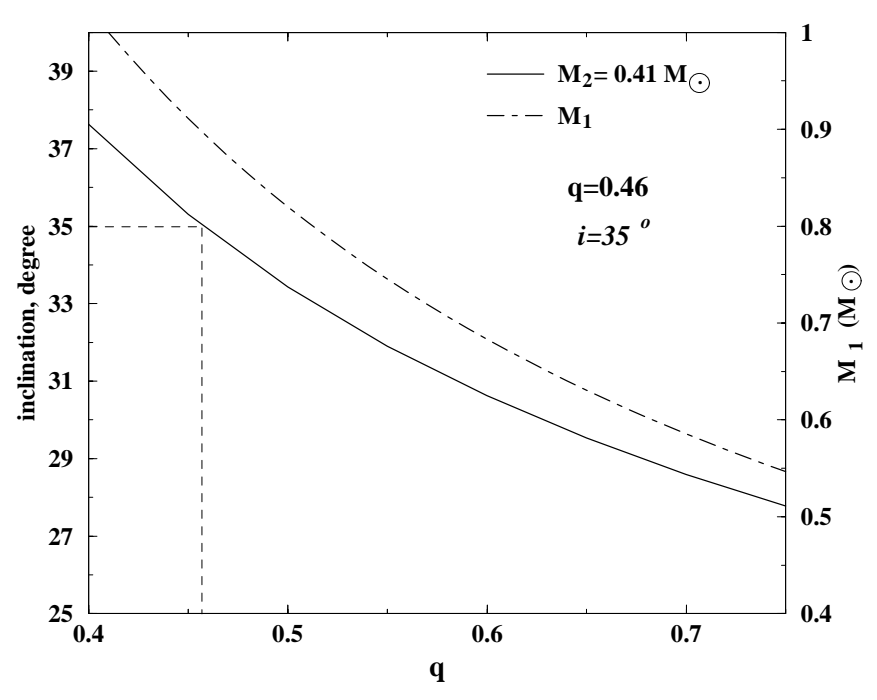

Fig. 6. The inclination angle of the system vs. $q=M_{2} / M_{1}$ is shown (solid line). The mass of WD in the system $M_{1}$ vs. $q$ for $M_{2}=0.41 M_{\odot}$ is given by the dot-dashed line. "Best fit" parameters from the secondary star position and the ballistic trajectory of the gas stream on the Doppler maps are marked

two iterations no further improvement was achieved. The diagnostic diagrams for $\mathrm{H}_{\beta}$ are shown in Fig. 3. Figure 4 (top) shows the $\mathrm{H}_{\beta}$ radial velocity curve.

A narrow single Gaussian profile was fitted to the prominent emission features in the profile of He II $\lambda 4686 \AA$, and the measured line centers were used to determine the radial velocity solution for 1 WGA J1958.2+3232. The radial velocity curve for He II $\lambda 4686$ line is presented in the middle panel of Fig. 4. These measurements were also subjected to the power spectrum analysis. The obtained orbital period is in good agreement with the values derived from the photometry and the $\mathrm{H}_{\beta}$ line radial velocities. The power spectra around the values corresponding to the orbital period from these three independent determinations are plotted in Fig. 5. One can see the excellent match of the central peak. We adopted $0.18152 \pm 0$ d 00011 as the final value for the orbital period of 1 WGA J1958.2+3232 from our observations. Longer time base observations are needed to improve this value.

Each of the radial velocity curves was fitted using a least-squares routine of the form

$v(t)=\gamma_{\mathrm{o}}+K_{1} * \sin \left(2 \pi\left(t-t_{\mathrm{o}}\right) / P\right)$,

where $\gamma_{\mathrm{o}}$ is the systematic velocity of the system, and $K_{1}$ is the semi-amplitude of radial velocity, both in $\mathrm{km} \mathrm{s}^{-1}$. The observation time is $t$, the epoch $t_{0}=2451761$ d. $2281 \pm$ 0 d 0001 corresponds to the \pm zero crossing of the $\mathrm{H}_{\beta}$ radial velocity curve, and therefore is a superior conjunction of the binary system (secondary located between observer and the WD). Accordingly the phase value at $t_{0}$ was set to 0.0 . Table 2 gives a summary of the radial velocity fits for $\mathrm{H}_{\beta}$ and $\mathrm{He}$ II 4686 emission line.

After refining the orbital period from spectroscopy, and determining the phase 0.0, the photometric light curve
Table 2. Radial velocity solution parameters

\begin{tabular}{ccc}
\hline \hline Name & $\begin{array}{c}\gamma_{\mathrm{o}} \\
\mathrm{km} \mathrm{s}^{-1}\end{array}$ & $\begin{array}{c}K_{1} \\
\mathrm{~km} \mathrm{~s}^{-1}\end{array}$ \\
\hline $\mathrm{H}_{\beta}$ & $-39 \pm 5$ & $74 \pm 7$ \\
HeII4686 & $-70.2 \pm 15.0$ & $197.4 \pm 25.7$ \\
\hline
\end{tabular}

was folded by the corresponding parameters and presented in the lower panel of Fig. 4.

Several conclusions can be made after considering the three curves in Fig. 4 in conjunction with and taking into account common knowledge of Intermediate Polar systems (Patterson 1994; Warner 1995):

- The double peaked Balmer lines are due to the presence of an accretion disk or ring orbiting the primary WD in this IP. RV variations in the wings of lines describe the rotation of the primary of the binary system;

- The S-wave present in the Balmer and HeII emission lines is evidence for a hot compact region on the accretion disk;

- From the difference of phases and amplitudes of radial velocities of the wings of $\mathrm{H}_{\beta}$ and the narrow component of He II we can conclude that the S-wave producing hot spot is located at $\approx 0.1-0.2$ phase ahead of the superior conjunction. It is difficult to estimate the location more precisely, because the matter in the spot has an intrinsic velocity. It is the usual location of the spot originating from the impact of the mass transfer stream with the accretion disk. However it is also the area which is most commonly heated by the energetic X-ray beam from the magnetically accreting pole on the surface of the WD in IPs (see the sketch in Hellier et al. 1989);

- The hot spot itself is eclipsed by the accretion ring as follows from the phasing of the light curve, the dips in the light curve centered at the phase $\approx 0.12 \phi_{\text {orb }}$. The primary at this phase starts to move toward the observer and the hot spot is on the opposite side of the ring approaching maximum velocity.

From our spectroscopic radial velocity solution, we can determine preliminary values for the basic system parameters of 1 WGA J1958.2+3232. First, from the mass-period and radius-period relations of Echevarría (1983)

$$
\begin{aligned}
M_{2} / M_{\odot} & =0.0751 P(h)^{1.16} \\
R_{2} / R_{\odot} & =0.101 P(h)^{1.05}, 1.4<P(h)<12
\end{aligned}
$$

we estimate the mass and radius of the secondary star as $M_{2}=0.41 M_{\odot}$ and $R_{2}=0.47 R_{\odot}$.

On the other hand, we can constrain the relation between inclination angle $i$ versus mass ratio $q$ :

$\sin ^{3}(i)=\frac{K_{1}^{3} P}{2 \pi G M_{2}}\left(\frac{q+1}{q}\right)^{2}$

if the mass ratio of the system is known (see Downes et al. 1986; Dobrzycka \& Howel 1992). The dependence of $i$ versus $q=M_{2} / M_{\mathrm{WD}}$ in the range 0.4 up to 0.75 is shown 

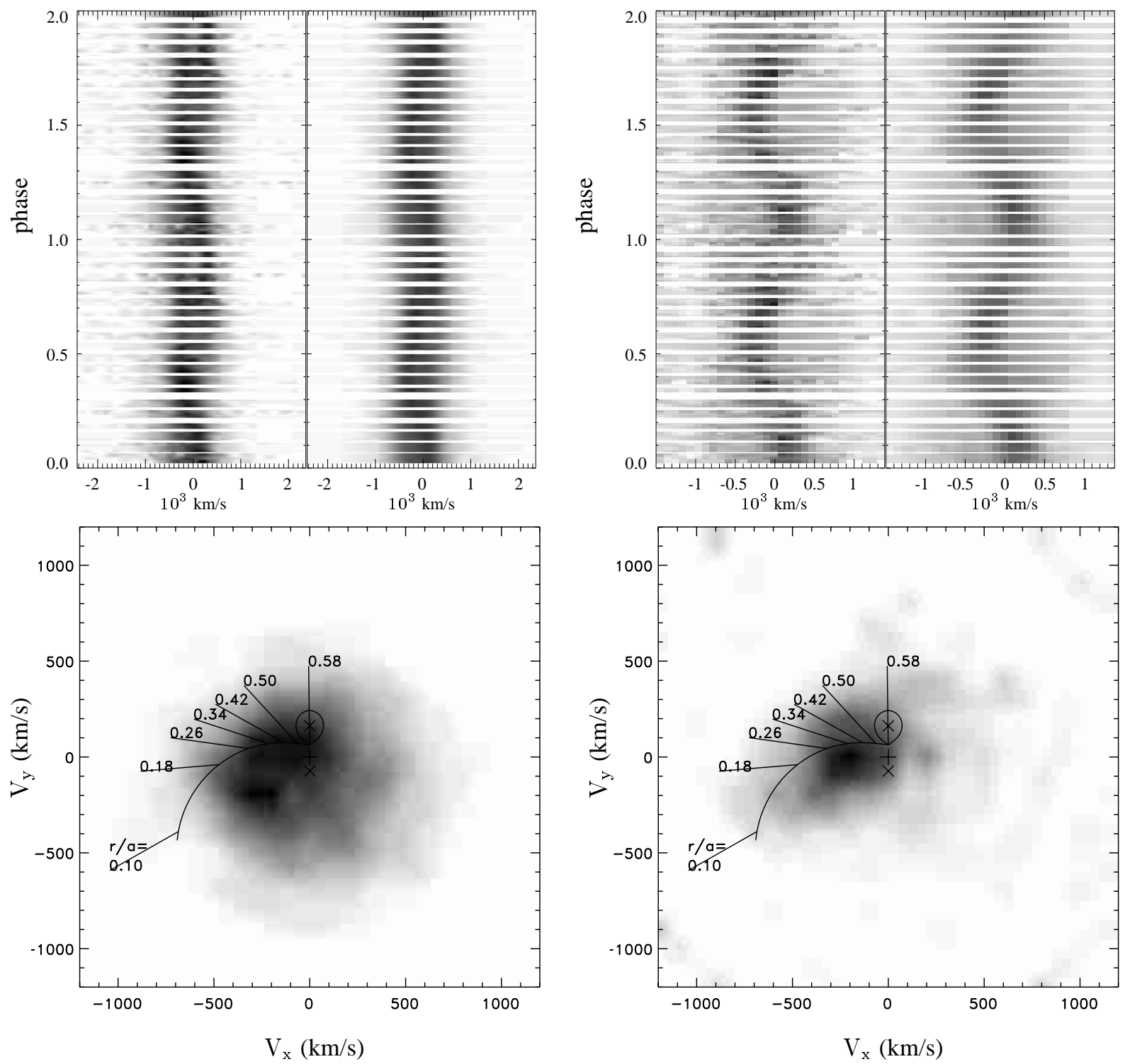

Fig. 7. Trailed, continuum-subtracted, spectra of 1 WGA J1958.2+3232 plotted in two cycles. Doppler maps of the emission lines $\mathrm{H}_{\beta}$ (left panel), He II (right panel) in velocity space $\left(V_{x}, V_{y}\right)$ are given. A schematic overlay marks the Roche lobe of the secondary, the ballistic trajectory and the magnetically funneled horizontal part of the accretion stream. The secondary star and gas-stream trajectory are plotted for $K=74 \mathrm{~km} \mathrm{~s}^{-1}$ and $q=0.46$

in Fig. 6 for the above determinated values of $K_{1 \mathrm{H}_{\beta}}, P_{\mathrm{orb}}$ and $M_{2}$.

Meanwhile, the mean mass estimate of 76 white dwarfs in $\mathrm{CVs}$ is $M_{\mathrm{WD}}=0.86 M_{\odot}$ (Sion 1999). Webbink (1990) gives statistically average white dwarf masses ratios $(q=0.29)$ and average masses for all systems $\left(M_{\mathrm{WD}}=\right.$ $\left.0.61 M_{\odot}\right)$ below the period gap and $\left(q=0.64, M_{\mathrm{WD}}=\right.$ $\left.0.82 M_{\odot}\right)$ above the period gap. Thus, the possible solutions lie in the narrow range of values.

We attempted to refine these values for 1 WGA J1958.2+3232 by constraining Doppler tomograms from observed emission line profiles. Doppler tomography is a useful tool to extract further information on CVs from trailed spectra. This method, which was developed by Marsh \& Horne (1988), uses the velocity
Table 3. The 1WGA1958.3+3232 adopted system parameters

\begin{tabular}{cccc}
\hline \hline Parameter & Value & Parameter & Value \\
\hline$P_{\text {orb }}$ & $0.18152 \mathrm{~d}$ & $R_{2}$ & $0.47 R_{\odot}$ \\
$P_{\text {rot }}$ & $733.7 \mathrm{~s}$ & $a$ & $1.5 R_{\odot}$ \\
$M_{2}$ & $0.41 M_{\odot}$ & $i$ & $35^{\circ}$ \\
$q$ & 0.46 & $M_{\mathrm{WD}}$ & $0.9 M_{\odot}$ \\
\hline
\end{tabular}

profiles of emission-lines at each phase to create a twodimensional intensity image in velocity-space coordinates $\left(V_{x}, V_{y}\right)$. Therefore, the Doppler tomogram can be interpreted as a projection of emitting regions in cataclysmic variables onto the plane perpendicular to the observers view. We used the code developed by Spruit (1998) to constrain Doppler maps of 1 WGA J1958.2+3232 with 


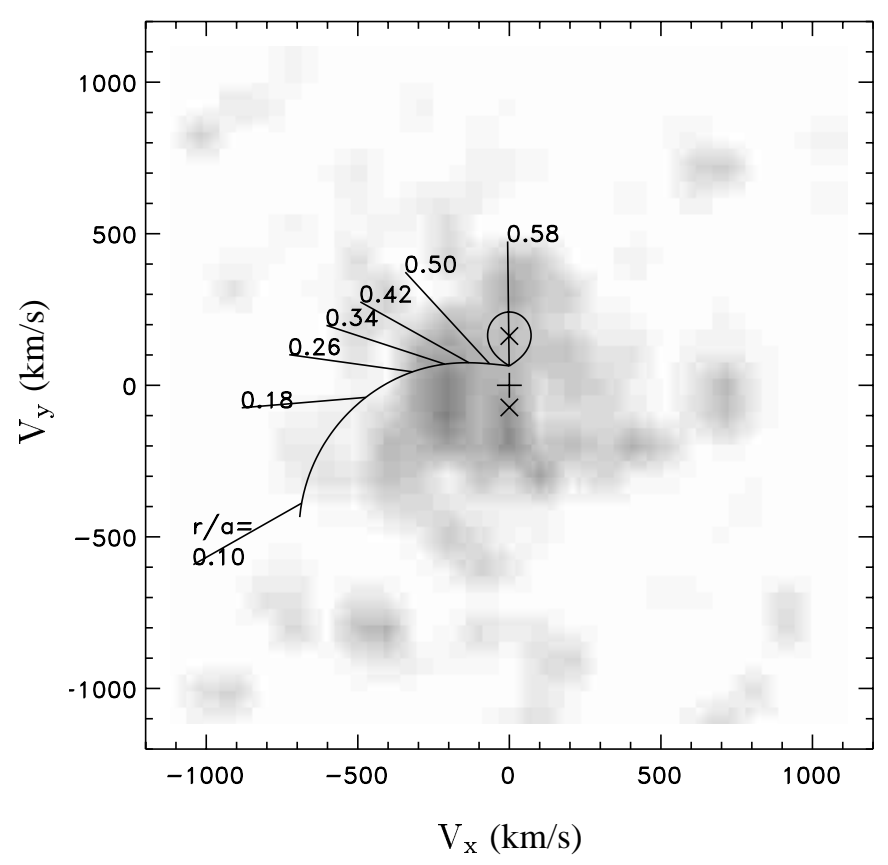

Fig. 8. Doppler maps of the emission line blend $\mathrm{C}$ III/N III in velocity space $\left(V_{x}, V_{y}\right)$ is given. A schematic overlay marks the Roche lobe of the secondary, the ballistic trajectory and the magnetically funneled horizontal part of the accretion stream. The secondary star and gas-stream trajectory are plotted for $K=74 \mathrm{~km} \mathrm{~s}^{-1}$ and $q=0.46$

the maximum entropy method. The resulting Doppler maps (or tomogram) of emission lines of $\mathrm{H}_{\beta}$, He II and the blend of $\mathrm{CIII} / \mathrm{N}$ III are displayed as a gray-scale image in Figs. 7 and 8. Also in Fig. 7 are displayed trailed spectra of $\mathrm{H}_{\beta}$ and He II in phase space and their corresponding reconstructed counterparts. Two features in the maps are distinct: an accretion disk seen as a dark circle extending to up to $\sim-700 \mathrm{kms}^{-1}$ on $\mathrm{H}_{\beta}$ doppler tomogram and a bright spot detected in all three maps to the left and below the center of mass at velocities $V_{x} \sim-225 \mathrm{~km} \mathrm{~s}^{-1}, V_{y} \sim-100 \mathrm{~km} \mathrm{~s}^{-1}$ in He II 4686. Apart from the spot a cometary tail linked to it and extending to $V_{x} \approx V_{y} \approx-500 \mathrm{~km} \mathrm{~s}^{-1}$ can be clearly seen on the He II map. These we identify with the mass transfer stream and its shape was essential for our selection of the ballistic trajectory. A help in interpreting Doppler maps are additional inserted plots which mark the position of the secondary star and the ballistic trajectory of the gas stream. Here we used our estimates of $P_{\text {orb }}$ and $M_{2}$ with various combinations of $i$ and $q$ from Fig. 6 in order to obtain the "best fit" (by simple eye inspection) of the calculated stream trajectory with the gray scale image. Our preferred solution for the inclination is $i=35^{\circ}$. It is marked in Fig. 6 and given in Table 3. Of course other close solutions are applicable. Comparing the location of spots in Doppler maps of He II and $\mathrm{H}_{\beta}$ we can actually distinguish two hot spots on the disk (Fig. 7). The elongated spot coinciding in both emission lines is probably caused by the mass transfer stream and the shock of impact with the disk, while the compact dense spot toward negative $V_{y}$ 's, seen much better in $\mathrm{H}_{\beta}$ than in the two other lines, is a result of heating of the disk by the X-ray beam. The C III/N III pattern mostly repeats that of He II, with lower intensity though (Fig. 8).

\section{Conclusions}

The 1 WGA J1958.2+3232 is found to be a "textbook" Intermediate Polar. It has an orbital period $P_{\text {orb }}=4.36$ above the period gap, as have the vast majority of IPs. It exhibits X-ray and optical coherent pulsations of the order of $\sim 0.05 P_{\text {orb }}$, undoubtedly originating from asynchronous spin of the magnetic WD in a close binary system. The beat period in optical light is also detectable. This is another characteristic of Intermediate Polars.

Other orbital parameters derived from the assumption that the system obeys the $P_{\text {orb }} \sim M_{2}$ relation for $\mathrm{CVs}$ also agree with accumulated data on other IPs and theoretical aspects (Warner 1995; Patterson 1994, see also URL ${ }^{3}$ ).

The radial velocity curves, the light curve and the Doppler tomography confirm the presence of an accretion ring around the WD and the existence of hot spots caused by heating of parts of the disk by the X-ray beam and from interaction with the mass transfer stream.

Acknowledgements. This work was supported in part by CONACYT project 25454-E and DGAPA project IN-118999.

\section{References}

Deeming, T. J. 1975, Ap\&SS, 36, 137

Dobrzycka, D., \& Howell, S. B. 1992, ApJ, 388, 614

Downes, R. A., Mateo, M., Szkody, P., Jenner, D. C., \& Margon, B. 1986, ApJ, 301, 240

Echevarría, J. 1983, Rev. Mex. Astron. Astrof., 8, 109

Hellier, C., Mason, K. O., \& Cropper, M. S. 1989, MNRAS, 237, 39P

Israel, G. L., Angelini, L., \& Campana, S. 1998, MNRAS, 248, 233

Israel, G. L., Covino, S., Polcaro, V. F., \& Stella, L. 1999, A\&A, 345, L1

Marsh, T. R., \& Horne, K. 1988, MNRAS, 235, 269

Negueruela, I., Reig, P., \& Clarl, J. S. 2000, A\&A, 354, L29

Patterson, J. 1994, PASP, 106, 209

Roberts, D. H., Lehar, J., \& Dreher, J. W. 1987, AJ, 93, 968

Rosen, S. R., Mason, K. O., \& Cordova, F. A 1988, MNRAS, 231, 549

Schneider, P. B., \& Young, P. 1980 ApJ, 238, 946

Shafter, A. W. 1983, ApJ, 267, 222

Sion, E. M. 1999, PASP, 111, 532

Spruit, H. C. 1998 [astro-ph/9806141]

Uslenghi, M., Bergamini, P., Catalano, S., Tommasi, L., \& Treves, A. 2000, A\&A, 359, 639

Warner, B. 1995, Cataclysmic Variable Stars (Cambridge Univ. Press, Cambridge)

Webbink, R. 1990, in Accretion-Powered Compact Binaries, ed. C. W. Mauche (Cambridge: Cambridge Univ. Press), 177

\footnotetext{
3 http://lheawww.gsfc.nasa.gov/users/mukai/

iphome/members.html\#cand
} 